\title{
Impacto da música na modulação autonômica em diferentes faixas etárias
}

\author{
Impact of music on autonomic modulation in different age groups \\ Impacto de la música en la modulación autónoma en diferentes grupos de edad
}

Fernando Seiji da Silva

ORCID: https://orcid.org/0000-0002-6373-8817

Universidade Federal do Triângulo Mineiro, Brasil

E-mail: fernando.silva@uftm.edu.br

Luciana Duarte Novais Silva

ORCID: https://orcid.org/0000-0002-1200-5680

Universidade Federal do Triângulo Mineiro, Brasil

E-mail: luciana.duarte.silva@uftm.edu.br

Jairo da Silva Custódio Júnior

ORCID: https://orcid.org/0000-0002-4572-2117

Universidade Federal do Triângulo Mineiro, Brasil

E-mail: jairoscjunior@gmail.com

Solon Quintão Henriques Júnior

ORCID: https://orcid.org/0000-0003-1804-6341

Universidade Federal do Triângulo Mineiro, Brasil

E-mail: solonquintao@gmail.com

Ana Paula Espindula

ORCID: https://orcid.org/0000-0002-9282-4482

Universidade Federal do Triângulo Mineiro, Brasil E-mail: ana.espindula@uftm.edu

Ricardo Luiz Smith

ORCID: https://orcid.org/0000-0001-8103-7371

Universidade Federal de São Paulo, Brasil

E-mail: rlsmith@unifesp.br

\begin{abstract}
Resumo
Objetivo: avaliar a influência dos diferentes tipos de músicas em diferentes faixas etária sobre a modulação autonômica da frequência cardíaca. Métodos: foram avaliados 96 voluntários, 44 no grupo musicalizado - GM (24,6 anos - 26 homens e 18 mulheres) e 52 no grupo não musicalizado - GNM (23,9 anos - 22 homens e 30 mulheres). Quatro músicas diferentes (M1, M2, M3 e M4) foram executadas por 22 minutos precedidos por cinco minutos de intervalo. A coleta de dados foi realizada po um monitor de frequência cardíaca, marca POLAR, modelo RS800CX. A análise dos dados foi feita no domínio do tempo pelos índices de RMSSD e pNN50, e no domínio da frequência pelos índices de baixa frequência - LF (atividade simpática), alta frequência - HF (atividade parassimpática) e LF/HF (balanço simpático-vagal). Foram utilizados testes estatístisticos para análise dos dados. Resultados: Para as diferentes faixas etárias no GM foram encontradas variâncias significativas entre os grupos de jovens e de meia idade para RMSSD (durante o repouso em M1 a M4), pNN50 (apenas em repouso), HF (durante o repouso, M1 e M2) e LF / HF (durante repouso, M1, M2 e M4), e os adultos comparados aos de meia-idade em repouso, M1 e M4. No GNM foram encontradas diferenças significativas comparado ao GM (maior em repouso em jovens do que de meia-idade e maior em jovens do que adultos e meia-idade durante M1 e M3) e BF / HF (maior em jovens em relação aos adultos e meiaidade durante a execução de M3 e M4). Conclusão: com o avançar da idade ocorre redução dos índices fisiológicos da variabilidade da frequência cardíaca. No GM esse resultado é mais evidente, pois os jovens deste grupo partem de valores maiores de índice de atividade parassimpática do que o GNM.
\end{abstract}

Palavras chave: Frequência cardíaca; Modulação autonômica; Música.

\section{Abstract}

Objective: to evaluate the influence of different types of music in different age groups on the autonomic modulation of heart rate. Methods: 96 volunteers were obtained, 44 in the musical group - GM (24.6 years - 26 men and 18 women) and 52 in the non-musicalized group - GNM (23.9 years - 22 men and 30 women). Four different songs (M1, M2, M3 and M4) were played for 22 minutes preceded by a five-minute break. Data collection was performed by a heart rate monitor, POLAR brand, model RS800CX. Data analysis was performed in the time domain by the RMSSD and pNN50 indices, and in the frequency domain by the low frequency - LF (sympathetic activity), high frequency HF (parasympathetic activity) and LF / HF (sympathetic-vagal balance) indices). Statistical tests were used for data analysis. Results: For the different age groups in the MG, variances were found between the young and middle-aged groups for RMSSD (during rest in M1 to M4), pNN50 (only at rest), HF (during rest, M1 and M2) and LF / HF 
(during rest, M1, M2 and M4), and adults compared to middle age at rest, M1 and M4. In GNM, differences were found related to GM (greater at rest in young people than middle-aged and greater in young people than adults and middle-aged during M1 and M3) and BF / HF (greater in young people compared to adults and a half age when performing M3 and M4). Conclusion: with advancing age, there is a reduction in the physiological indices of heart rate variability. In GM, this result is more evident, as young people in this group start from higher values of parasympathetic activity index than in the GNM.

Keywords: Heart rate; Autonomic modulation; Song.

\begin{abstract}
Resumen
Objetivo: evaluar la influencia de diferentes tipos de música en diferentes grupos de edad sobre la modulación autonómica de la frecuencia cardíaca. Métodos: se obtuvieron 96 voluntarios, 44 en el grupo musical - GM (24,6 años - 26 hombres y 18 mujeres) y 52 en el grupo no musicalizado - GNM (23,9 años - 22 hombres y 30 mujeres). Se tocaron cuatro canciones diferentes (M1, M2, M3 y M4) durante 22 minutos precedidos de un descanso de cinco minutos. La recolección de datos se realizó mediante un monitor de frecuencia cardíaca, marca POLAR, modelo RS800CX. El análisis de los datos se realizó en el dominio del tiempo por los índices RMSSD y pNN50, y en el dominio de la frecuencia por los índices de baja frecuencia - LF (actividad simpática), alta frecuencia - HF (actividad parasimpática) y LF / HF (equilibrio simpático-vagal). Se utilizaron pruebas estadísticas para el análisis de datos. Resultados: Para los diferentes grupos de edad en la MG, se encontraron variaciones entre los grupos de jóvenes y de mediana edad para RMSSD (durante el reposo en M1 a M4), pNN50 (solo en reposo), IC (durante el reposo, M1 y M2) y LF / HF (en reposo, M1, M2 y M4), y adultos frente a mediana edad en reposo, M1 y M4. En GNM se encontraron diferencias relacionadas con GM (mayor en reposo en jóvenes que en mediana edad y mayor en jóvenes que en adultos y de mediana edad durante M1 y M3) y BF / HF (mayor en jóvenes en comparación con adultos y una media edad al realizar M3 y M4). Conclusión: con el avance de la edad se observa una reducción de los índices fisiológicos de variabilidad de la frecuencia cardíaca. En GM, este resultado es más evidente, ya que los jóvenes de este grupo parten de valores más altos del índice de actividad parasimpática que en el GNM.
\end{abstract}

Palabras clave: Frecuencia cardiaca; Modulación autónoma; Canción.

\title{
1. Introdução
}

O prazer na música surge a partir de interações entre circuitos corticais que permitem que previsões e expectativas surjam a partir de padrões de som e sistemas subcorticais responsáveis pela recompensa e valorização (Zatorre e Salimpoor, 2013). A dissonância recruta regiões paralimbicas e o córtex auditivo, sendo que as regiões paralimbicas geram uma resposta emocional para as dissonâncias, enquanto o córtex auditivo está envolvido com a análise perceptiva (Gosselin et al., 2006). De acordo com esta análise, uma lesão no córtex auditivo levaria a uma deficiência na percepção da dissonância resultando em déficit no processamento emocional do estímulo (Peretz et al., 2001).

$\mathrm{O}$ ato de escutar música facilita a neurogênese, a reparação e a regeneração dos nervos cerebrais, pois ajusta a secreção de hormônios esteróides, o que aumenta a plasticidade cerebral (Fukui, Toyoshima, 2008). A música afeta os níveis destes hormônios, tais como cortisol, testosterona e estrogênio, através da via auditiva, na área auditiva, particularmente pelo circuito emocional mediado pelo sistema límbico, assim como afeta os genes receptores dessas substâncias. (Fukui, Toyoshima, 2008; Silva et al., 2021). A música é uma ferramenta importante e necessária para o desenvolvimento dos aspectos cognitivos, linguísticos, psicomotores e sócio-afetivos (Santos, Carvalho \& Selva, 2020).

Estudo com músicos profissionais, músicos amadores e indivíduos não musicalizados, a partir de imagens de ressonâncias magnéticas de alta resolução constatou que existe um aumento significante no volume da massa cinzenta nas regiões motora, auditiva e visual-espacial do cérebro dos músicos profissionais em relação aos demais grupos. Acredita-se que esta diferença no volume pode ter ocorrido devido a uma resposta adaptativa à aquisição das habilidades a longo prazo e os treinos repetitivos de tais habilidades musicais (Gaser \& Schlaug, 2003). Ainda, no estudo de Iwanaga et al. (2005), foi observado que tanto a música relaxante quanto o silêncio induziam a um aumento do relaxamento e consequentemente a diminuição da tensão, no entanto a música excitativa diminuía a percepção de tensão e aumentava a percepção de relaxamento com o aumento do número de sessões. A baixa frequência e a razão $\mathrm{BF} / \mathrm{AF}$ aumentaram durante as músicas, sedativa e excitativa, mas diminuíram na seção em silêncio; o componente de alta frequência foi maior durante a música sedativa e o 
silêncio do que durante a música excitativa, indicando que esta diminui a ação do componente parassimpático (Iwanaga et al., 2005).

De acordo com os estudos apresentados acima é evidente o efeito da música sobre o sistema nervoso autônomo e consequentemente sobre a variabilidade da frequência cardíaca. No entanto é necessário elucidar se as respostas são diferentes em relação aos diferentes tipos de estímulo musical, bem como a condição do indivíduo estudado em relação a diferentes faixas de idade e se musicalizado ou não. Assim o objetivo do presente estudo foi avaliar a influência dos diferentes tipo de música em diferentes faixas etária sobre a modulação autonômica da frequência cardíaca. Tendo como hipótese que os diferentes tipos de música leva a diferentes modulações autonômicas da frequencia cardíaca em faixas etárias diversas.

\section{Metodologia}

Estudo quantitativo, experimental prospectivo controlado, cego (Pereira et al., 2018), desenvolvido no Laboratório de Cardiologia, no Centro Educacional da Universidade Federal do Triângulo Mineiro (UFTM), no município de Uberaba, MG, aprovado pelos Comitês de Ética em Pesquisa da UFTM e da Universidade Federal de São Paulo (UNIFESP), sob parecer da Universidade Federal dos Vales do Jequitinhonha e Mucuri (UFVJM) e validado pela UFTM sob nº 012/09.

Todos os voluntários foram informados sobre os procedimentos experimentais aos quais seriam submetidos, do caráter não invasivo dos testes, bem como no fato destes não afetarem sua saúde. Os indivíduos que concordaram em participar assinaram o termo de consentimento livre e esclarecido, de acordo com as normas do Conselho Nacional de Saúde (196/96).

Foram avaliados 96 voluntários: 44 musicalizados (GM), (idade média 24,6 anos, 26 homens e 18 mulheres); e 52 não musicalizados (GNM), (idade média de 23,9 anos, 22 homens e 30 mulheres), Sendo o GM e GNM subdivididos em: Jovens (indivíduos até 18 anos de idade); adultos (indivíduos com idade entre 18 e 39 anos) e meia idade (indivíduos com idade entre 40 e 60 anos).

Os critérios de exclusão foram os voluntários que apresentassem diagnósticos ou relato de distúrbios auditivos, doenças cardiovasculares ou pulmonares, fumantes e os que faziam uso de betabloqueadores, diuréticos, bloqueadores de canal de cálcio e inibidores de enzima conversora de angiotensina e antidepressivos. Não foram incluídos nesse estudo os indivíduos que haviam realizado terapia de realidade virtual previamente, impossibilitados de permanecer na posição ortostática, com comprometimento visual grave ou não compensado com uso de lentes corretivas e crianças com distúrbios ortopédicos que resultavam em quadro álgico. Como critérios de inclusão para o GM os voluntários deveriam ser estudantes do Conservatório Estadual de Música Renato Frateschi, estudando algum tipo de instrumento musical há no mínimo um ano, sem interrupção. Para o GNM os voluntários não poderiam ter realizado qualquer tipo de estudo ou treinamento musical.

Os experimentos foram realizados no mesmo período do dia para reduzir as influências das variações circadianas sobre o organismo. Foi recomendado que os voluntários comparecessem com roupas e calçados confortáveis, que não fizessem uso de bebidas alcoólicas e/ou estimulantes 24 horas antes dos testes, que fizessem uma refeição leve pelo menos duas horas antes do teste e que não realizassem atividade física extenuante no dia anterior.

Para reduzir a ansiedade e expectativa por parte dos voluntários, foram feitos procedimentos de familiarização dos indivíduos com o protocolo de teste, com a equipe de pesquisadores e equipamentos, no mínimo uma semana antes dos protocolos experimentais. Os protocolos foram realizados sempre pelos mesmos avaliadores, nos dois grupos estudados. Com o mesmo intuito, foi mantido um trânsito mínimo de pessoas no laboratório durante a execução dos experimentos.

Para a avaliação da modulação autonômica da resposta da frequência cardíaca a partir de sua variabilidade (VFC) durante o repouso em silêncio e sob estímulo musical, foi utilizado um monitor de frequência cardíaca, da marca POLAR, modelo RS800CX para obtenção dos dados de FC e posterior análise da VFC. Primeiramente foi feito o posicionamento da cinta no tórax (na altura do coração) e do relógio no pulso do voluntário, além do fone de ouvido. A partir de então, os 
voluntários foram orientados a manter-se em repouso na posição sentada, com uma respiração tranquila e evitando conversar com os avaliadores. Após 10 minutos, para estabilização das variáveis cardiorrespiratórias, foi iniciada a coleta dos dados de frequência cardíaca de repouso por 5 minutos e trinta segundos, na posição sentada em uma sala com o ambiente silencioso. Depois desse período, os voluntários foram expostos a um período de 22 minutos de música, mantendo a mesma coleta de FC. Foram impostas quatro músicas com ritmos diferentes durante 5 minutos e trinta segundos cada uma (Tabela 1).

Tabela 1. Música impostas durante as avaliações.

\begin{tabular}{ccccc}
\hline Música & Nome e estilo musical & Estilo musical & Tempo & Adquirida \\
\hline 1 & Terceira Sinfonia (Heróica), de Beethoven & Clássica, & 5 minutos e & Via Itunes \\
& & instrumental, ritmo & 30 & \\
& & lento & segundos & \\
2 & Day Ligth Daylight of Konoha, de Toshiro Masuda & Instrumental, ritmo & 5 minutos e & Via Itunes \\
& & lento & 30 & \\
& & segundos & Via Itunes \\
3 & Bad Romance, de Lady Gaga & Pop, vozes, ritmo & 5 minutos e & rápido \\
& & 30 segundos & \\
4 & Drum Solos, de Kitaro & Instrumental & 5minutos e & Via Itunes \\
& & percussão ritmo & 30 & \\
& & lento & segundos & \\
\hline
\end{tabular}

Fonte: Autores.

Após a coleta da FC, foram descartados os 15 segundos iniciais e finais de cada amostra, a fim de selecionar trechos mais estáveis de sinal. Resultando em um tempo total de 5 minutos de análise em cada condição, totalizando 25 minutos por voluntário. Seguido por uma inspeção visual da distribuição dos dados de FC coletados, para verificar a estabilidade do traçado obtido, bem como a ausência de artefatos, para a realização das análises no domínio do tempo e da frequência, fornecidos pelo software Polar Precision Performance Sw, que acompanha o monitor de FC.

Para a análise dos dados no domínio do tempo foram utilizados os índices RMSSD dos intervalos R-R (iR-R) e pNN50. De acordo com Antila (1979), o índice RMSSD, corresponde a raiz quadrada da somatória do quadrado das diferenças entre os iR-R no registro divididos pelo número de iR-R em um tempo determinado menos um iR-R. Já o pNN50 representa a proporção de pares de intervalos RR normais consecutivos, cuja diferença é maior ou igual a $50 \mathrm{~ms}$ em relação a todo o registro.

Por meio da análise espectral foram obtidas as bandas de muito baixa frequência, de baixa frequência (BF), e de alta frequência (AF). Em nosso estudo utilizamos duas faixas de frequência que melhor representam a atuação dos componentes vagal e simpático no controle da $\mathrm{FC}$, ou seja, faixa $\mathrm{BF}$, correspondendo de 0,04 a $0,15 \mathrm{~Hz}$ e a faixa de $\mathrm{AF}$, que corresponde de 0,15 a $0,4 \mathrm{~Hz}$. Além dos valores absolutos de $\mathrm{BF}$ e AF, tais componentes também foram expressos como a razão entre as áreas absolutas de baixa e alta frequência (razão BF/AF), que é indicativo do balanço simpatovagal.

Após a verificação da distribuição Gaussiana dos dados, foram escolhidos testes estatísticos paramétricos. Foram realizadas comparações intragrupos entre as condições de silêncio e os diferentes tipos de música dos valores médios dos índices RMSSD e pNN50 no domínio do tempo. Já no domínio da frequência, foram utilizadas a razão BF/AF, BF e AF. O teste estatístico utilizado foi One-way ANOVA, com post-hoc de Tukey, por meio do software GraphPad Instat, versão 3, 1997. Para a comparação intergrupos, foi utilizado o teste t não pareado comparando os valores médios dos índices RMSSD e pNN50, no domínio do tempo. O mesmo teste foi utilizado para a comparação dos índices no domínio da frequência: AF, BF e 
a razão $\mathrm{BF} / \mathrm{AF}$. A comparação intergrupos foi utilizada para comparar indivíduos musicalizados e não musicalizados em cada faixa etária. Todos os testes estatísticos utilizados neste estudo tiveram nível de significância estabelecido em 5\%.

\section{Resultados}

A análise no domínio do tempo no GM, pode-se observar valores do índice RMSSD significativamente maiores em relação ao subgrupo meia idade tanto no repouso quanto nos diferentes tipos de música. E do índice pNN50 durante o repouso. Já no GNM não foram encontradas diferenças estatisticamente significativas dos valores dos índices do domínio do tempo no repouso e durante a execução das músicas nas faixas etárias avaliadas (Tabela 2 e 3).

A análise no domínio da frequência no GM demonstrou valores estatisticamente maiores da banda AF no subgrupo jovem em relação ao subgrupo meia idade no repouso e durante a execução das músicas 1 e 2, sendo que a os valores da banda $\mathrm{BF}$ não foram estatisticamente diferentes entre as faixas etárias. Quando se comparou a razão $\mathrm{BF} / \mathrm{AF}$ foi encontrada diferença estatisticamente significante entre o subgrupo jovem e meia idade no repouso e na execução das músicas 1, 2 e 4, com maiores valores para o subgrupo de meia idade. Da mesma forma, os valores da razão $\mathrm{BF} / \mathrm{AF}$ também foram significativamente maiores para o subgrupo meia idade em relação ao subgrupo adulto, durante o repouso e na execução das músicas 1 e 4 (Tabela 2 ).

No GNM, os valores da banda de BF foram significativamente maiores para os jovens em relação ao subgrupo meia idade durante o repouso e na execução das músicas 1 e 3 . Da mesma forma, os jovens também apresentaram maiores valores de BF em relação ao subgrupo adulto, durante as músicas 1 e 3. Quando se avaliou a razão BF/AF foi observado valores significativamente maiores no subgrupo jovem em relação aos subgrupos adulto e meia idade durante a execução das músicas 3 e 4 (Tabela 3). 
Tabela 2. Valores dos índices no domínio do tempo (RMSSD e pNN50) e no domínio da frequência (BF, $A F, B F / A F)$ dos voluntários musicalizados $(n=44)$, subdivididos em três faixas etárias.

\begin{tabular}{|c|c|c|c|c|}
\hline & & Jovens & Adultos & Meia idade \\
\hline \multirow{5}{*}{$\sum_{\tilde{\simeq}}^{\mathscr{D}}$} & Repouso & $40,0 \pm 19,6^{+}$ & $37,7 \pm 18,0$ & $15,8 \pm 8,4$ \\
\hline & M1 & $37,4 \pm 22,7^{+}$ & $30,0 \pm 15,1$ & $11,6 \pm 8,3$ \\
\hline & M2 & $35,7 \pm 21,1^{+}$ & $28,8 \pm 13,8$ & $14,4 \pm 5,6$ \\
\hline & M3 & $34,3 \pm 17,1^{+}$ & $29,1 \pm 15,1$ & $15,6 \pm 4,7$ \\
\hline & M4 & $35,0 \pm 19,6^{+}$ & $28,8 \pm 16,1$ & $13,7 \pm 6,0$ \\
\hline \multirow{5}{*}{ 畐 } & Repouso & $9,6 \pm 7,8^{+}$ & $9,0 \pm 6,8$ & $1,2 \pm 1,2$ \\
\hline & M1 & $8,4 \pm 9,1$ & $4,9 \pm 4,3$ & $0,3 \pm 0,7$ \\
\hline & M2 & $7,4 \pm 8,5$ & $4,9 \pm 4,8$ & $0,7 \pm 0,9$ \\
\hline & M3 & $7,1 \pm 7,7$ & $4,8 \pm 4,7$ & $0,6 \pm 0,7$ \\
\hline & M4 & $7,0 \pm 7,8$ & $4,7 \pm 5,5$ & $0,3 \pm 0,4$ \\
\hline \multirow{5}{*}{$\frac{1}{n}$} & Repouso & $25,2 \pm 7,5$ & $28,9 \pm 8,6$ & $26,5 \pm 15,8$ \\
\hline & M1 & $27,8 \pm 10,3$ & $29,8 \pm 5,8$ & $24,9 \pm 12,2$ \\
\hline & M2 & $27,6 \pm 7,6$ & $31,3 \pm 6,7$ & $30,0 \pm 14,3$ \\
\hline & M3 & $30,7 \pm 6,8$ & $30,7 \pm 7,0$ & $28,7 \pm 7,0$ \\
\hline & M4 & $25,5 \pm 6,4$ & $26,7 \pm 6,9$ & $25,4 \pm 7,8$ \\
\hline \multirow{5}{*}{$\frac{1}{4}$} & Repouso & $21,4 \pm 9,9^{+}$ & $17,5 \pm 9,8$ & $7,8 \pm 5,7$ \\
\hline & M1 & $22,1 \pm 12,1^{+}$ & $15,9 \pm 11,8$ & $5,3 \pm 3,3$ \\
\hline & M2 & $17,6 \pm 10,0^{+}$ & $12,0 \pm 6,1$ & $7,3 \pm 4,0$ \\
\hline & M3 & $15,6 \pm 8,4$ & $11,9 \pm 6,5$ & $7,9 \pm 3,6$ \\
\hline & M4 & $16,3 \pm 8,8$ & $13,7 \pm 6,7$ & $7,5 \pm 7,5$ \\
\hline \multirow{5}{*}{$\sum_{\frac{1}{x}}^{\frac{5}{4}}$} & Repouso & $1,5 \pm 0,9^{+}$ & $2,1 \pm 1,2^{+}$ & $5,3 \pm 4,7$ \\
\hline & M1 & $1,7 \pm 1,3^{+}$ & $3,0 \pm 1,8^{+}$ & $6,1 \pm 4,6$ \\
\hline & M2 & $2,2 \pm 1,8^{+}$ & $3,2 \pm 1,5$ & $5,9 \pm 5,1$ \\
\hline & M3 & $2,7 \pm 1,9$ & $3,2 \pm 1,6$ & $5,2 \pm 4,6$ \\
\hline & M4 & $2,0 \pm 1,3^{+}$ & $2,3 \pm 0,9^{+}$ & $7,3 \pm 5,9$ \\
\hline
\end{tabular}

*Diferença significativa em relação ao grupo Adultos. +Diferença significativa em relação ao grupo Meia Idade. Nível de significância: $\mathrm{p}<0,05$. Fonte: Autores. 
Tabela 3. Valores dos índices no domínio do tempo (RMSSD e pNN50) e no domínio da frequência (BF, $A F, B F / A F)$ dos voluntários não musicalizados $(\mathrm{n}=52)$, subdivididos em três faixas etárias.

\begin{tabular}{|c|c|c|c|c|}
\hline & & Jovens & Adultos & Meia idade \\
\hline \multirow{5}{*}{$\underset{\mathscr{\Omega}}{\mathscr{\mathscr { A }}}$} & Repouso & $32,2 \pm 12,9$ & $41,6 \pm 23,7$ & $19,9 \pm 8,9$ \\
\hline & M1 & $28,4 \pm 11,7$ & $41,5 \pm 26,1$ & $20,5 \pm 11,4$ \\
\hline & M2 & $25,5 \pm 10,1$ & $39,5 \pm 26,0$ & $20,7 \pm 14,1$ \\
\hline & M3 & $22,7 \pm 8,0$ & $37,7 \pm 25,4$ & $19,5 \pm 12,5$ \\
\hline & M4 & $22,9 \pm 9,6$ & $36,5 \pm 25,4$ & $18,5 \pm 11,1$ \\
\hline \multirow{5}{*}{ 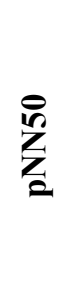 } & Repouso & $7,28 \pm 5,5$ & $9,5 \pm 9,6$ & $1,45 \pm 2,6$ \\
\hline & M1 & $4,94 \pm 4,3$ & $9,6 \pm 10,7$ & $1,7 \pm 3,9$ \\
\hline & M2 & $3,39 \pm 3,1$ & $8,7 \pm 10,2$ & $2,85 \pm 6,6$ \\
\hline & M3 & $2,45 \pm 2,3$ & $7,7 \pm 9,4$ & $1,7 \pm 3,7$ \\
\hline & M4 & $3,12 \pm 3,7$ & $7,3 \pm 9,6$ & $1,5 \pm 3,4$ \\
\hline \multirow{5}{*}{$\frac{5}{\infty}$} & Repouso & $30,6 \pm 7,7^{+}$ & $24,0 \pm 8,9$ & $18,1 \pm 5,4$ \\
\hline & M1 & $33,6 \pm 8,7^{*}$, & $23,4 \pm 8,5$ & $21,9 \pm 5,0$ \\
\hline & M2 & $31,4 \pm 9,9$ & $24,4 \pm 11,0$ & $21,7 \pm 4,5$ \\
\hline & M3 & $33,6 \pm 8,2^{*}$, & $23,5 \pm 9,4$ & $22,3 \pm 5,2$ \\
\hline & M4 & $32,0 \pm 11,0$ & $24,5 \pm 10,0$ & $22,5 \pm 6,4$ \\
\hline \multirow{5}{*}{$\frac{5}{4}$} & Repouso & $17,4 \pm 11,6$ & $18,4 \pm 9,8$ & $12,05 \pm 13,3$ \\
\hline & M1 & $15,0 \pm 8,4$ & $19,3 \pm 12,0$ & $18,8 \pm 12,3$ \\
\hline & M2 & $12,1 \pm 6,0$ & $14,4 \pm 10,2$ & $12,1 \pm 6,8$ \\
\hline & M3 & $9,7 \pm 5,1$ & $14,1 \pm 8,4$ & $13,6 \pm 5,1$ \\
\hline & M4 & $9,0 \pm 4,6$ & $15,7 \pm 10,8$ & $11,4 \pm 4,5$ \\
\hline \multirow{5}{*}{$\frac{2}{x}$} & Repouso & $2,7 \pm 1,9$ & $2,0 \pm 2,1$ & $2,5 \pm 1,2$ \\
\hline & M1 & $2,9 \pm 1,7$ & $1,9 \pm 1,3$ & $1,6 \pm 0,9$ \\
\hline & M2 & $3,5 \pm 2,6$ & $2,1 \pm 1,6$ & $2,2 \pm 0,9$ \\
\hline & M3 & $4,6 \pm 3,2^{*}$ & $2,2 \pm 1,4$ & $1,9 \pm 0,9$ \\
\hline & M4 & $4,9 \pm 3,6^{*}$ & $2,3 \pm 1,5$ & $2,2 \pm 0,8$ \\
\hline
\end{tabular}

*Diferença significativa em relação ao grupo Adultos. +Diferença significativa em relação ao grupo Meia Idade. Nível de significância: $p<0,05$. Fonte: Autores.

\section{Discussão}

O presente estudo teve como objetivo avaliar a modulação autonômica da frequência cardíaca sob o efeito de estímulo musical em indivíduos musicalizados e não musicalizados e a influência dos diferentes tipo de música em diferentes faixas etárias sobre a modulação autonômica da frequência cardíaca. Sendo nossa hipótese validada.

Estudos têm mostrado que as respostas fisiológicas dos sistemas orgânicos em repouso são influenciadas por vários fatores, tais como: idade, sexo, características antropométricas, condição de saúde e fatores ligados às condições ambientais 
(Gallo Jr et al., 1995; Barbosa et al., 1996; Davy et al., 1998; Schuit et al., 1999; Angelink et al., 2001; Catai et al., 2002; Silva et al., 2021).

Em nosso estudo as diferentes faixas etárias no grupo musicalizado, demostrou diferença estatisticamente significante nos índices RMSSD em repouso e durante a execução de todas as músicas em indivíduos jovens em relação aos de meia idade. Em relação ao pNN50, a diferença significativa foi observada na condição de repouso. Já nos índices do domínio da frequência, foram observadas diferenças estatisticamente significantes na banda de alta frequência (AF), no repouso e durante a execução das músicas 1 e 2 de jovens em relação a meia idade. Em relação ao balanço simpato-vagal, as diferenças significantes observadas foram dos jovens em relação à meia idade, exceto durante a execução da música 3 e de adultos em relação aos indivíduos de meia idade nas condições de repouso, nas música 1 e 4 . A mesma comparação por faixas etárias foi feita nos indivíduos não musicalizados, sendo que neste grupo não foi observada diferença estatisticamente significante nos índices no domínio do tempo. Já no domínio da frequência foram observadas diferenças significantes no índice BF e na razão $\mathrm{BF} / \mathrm{AF}$. Em relação à banda de $\mathrm{BF}$ os valores foram significativamente maiores nos jovens em relação aos indivíduos de meia idade durante o repouso e maiores nos jovens em relação aos adultos e aos de meia idade durante a música 1 e a música 3 . O balanço simpato-vagal avaliado pela razão $\mathrm{BF} / \mathrm{AF}$ foi maior nos indivíduos jovens em relação aos indivíduos adultos e de meia idade durante a execução das músicas 3 e 4 . O presente resultado corrobora estudos prévios na literatura que demonstram redução dos índices da VFC com o avançar da idade, demonstrando principalmente diminuição do tônus vagal. Isso se deve ao fato de que em jovens ocorre maior responsividade autonômica, associado ao fato de que nestes grupos a integridade do sistema neurocárdico encontra-se preservada (Zuttin et al., 2008; Paschoal et al., 2006).

$\mathrm{Na}$ literatura pesquisada encontra-se descrito que a musicoterapia oferecida a pacientes submetidos a ressonância magnética tem se mostrado uma intervenção eficaz para alcançar uma mudança específica no estado fisiológico, emocional e comportamental (Nieto-Romero, 2017). Em outro estudo que objetivou avaliar os efeitos da música sobre o estado de ansiedade, parâmetros fisiológicos e laboratoriais, em doadores de sangue, a musica não foi capaz de reduziu os níveis do estado de ansiedade. Contudo, foi possível demonstrar a eficácia da intervenção na redução da frequência cardíaca, frequência respiratória e níveis de cortisol sanguíneo, os quais apresentam-se alterados frente a situações ansiogênicas (Silva et al., 2021). Em outro estudo que avaliou o impacto da música como redutor de ansiedade no atendimento odontológico de crianças, demonstrou redução significante na frequência cardíaca nas crianças que escutaram música durante o atendimento odontológico. No grupo sem música, a frequência cardíaca manteve-se inalterada durante todo o atendimento (Tshiswaka \& Pinheiro, 2020). Outro estudo avaliou o efeito de uma intervenção musical sobre a ansiedade e parâmetros vitais em 60 pacientes com idade igual ou superior a 18 anos com doença renal crônica em comparação ao cuidado de clínicas de hemodiálise, concluiu que o estímulo musical infere ter potencial de intervenção para a redução do estado de ansiedade durante sessões de hemodiálise (Melo et al., 2018). Já em outro estudo que avaliou o efeito agudo da música heavy metal na regulação autonômica cardíaca em mulheres adultas jovens entre 18 e 30 anos expostas a músicas "pesadas" (75-84 dB) por 20 min, sugeriu que a estimulação musical aguda com música "pesada" influencia a regulação autonômica cardíaca em um nível mais complexo sendo benéfico para a função cardíaca (Nogueira et al., 2015).

Assim podemos inferir que a música é uma alternativa não farmacológica que pode reduzir os níveis de ansiedade em diferentes faixas etárias.

\section{Conclusão}

Com o avançar da idade ocorre a redução fisiológica dos indices da VFC, sendo que no grupo musicalizado esse resultado fica mais evidenciado, já que os jovens deste grupo partem de valores de índices de atividade parassimpática maiores 
do que os não musicalizados. Sugerimos para futuras pesquisas um acompanhamento ao longo do tempo para verificar a permanencia ou não dos resultados apresentados, assim como a avaliação em diferentes situações de ansiedade.

\section{Agradecimentos}

Aos servidores da disciplina de Anatomia Humana da UFTM; aos professores e alunos do Conservatório Estadual de Música Renato Frateschi, por todo o apoio ao projeto e a FUNEPU, pelo apoio financeiro.

\section{Referências}

Agelink, M. W., Malessa, R., Baumann, B., Majewski, T., Akila, F., Zeit, T., \& Ziegler, D. (2001). Standardized tests of heart rate variability: normal ranges obtained from 309 healthy humans, and effects of age, gender, and heart rate. Clin Auton Res. 11(2), 99-108.

Antila K. (1979)Quantitative characterization of heart rate during exercise. Scand J Clin Lab Invest Suppl. 153 , 3-68.

Barbosa, P. R., Barbosa Filho, J., \& de Sá, C.A. (1996). Effects of age, sex and coronary heart disease on the autonomic modulation of the heart. Arq Bras Cardiol. 67(5), 325-9.

Catai, A. M., Chacon-Mikahil, M. P., Martinelli, F. S., Forti, V.A., Silva, E., Golfetti, R., Martins, L. E., Szrajer, J. S., Wanderley, J. S., Lima-Filho, E. C., Milan, L. A., Marin-Neto, J. A., Maciel, B. C., Gallo-Junior, L. (2002). Effects of aerobic exercise training on heart rate variability during wakefulness and sleep and cardiorespiratory responses of young and middle-aged healthy men. Braz J Med Biol Res. 35(6), 741-52.

Davy, K. P., DeSouza, C. A., Jones, P. P., \& Seals, D. R. (1998). Elevated heart rate variability in physically active young and older adult women. Clin Sci (Lond). 94(6), 579-84.

Fukui, H., \&Toyoshima, K. (2008). Music facilitate the neurogenesis, regeneration and repair of neurons. Med Hypotheses. 71(5),765-9.

Gallo, L, Jr., Maciel, B. C., Marin-Neto, J. A., Martins, L. E., Lima-Filho, E. C., Golfetti, R., Chacon, M. P., \& Forti, V. A. (1995). Control of heart rate during exercise in health and disease. Braz J Med Biol Res. 28(11-12), 1179-84.

Gaser, C., \& Schlaug, G. (2003). Brain structures differ between musicians and non-musicians. J Neurosci. 23(27), 9240-5.

Gosselin, N., Samson, S., Adolphs, R., Noulhiane, M., Roy, M., Hasboun, D., Baulac, M., Peretz, I. (2006). Emotional responses to unpleasant music correlates with damage to the parahippocampal cortex. Brain. 129(10), 2585-92.

Iwanaga, M., Kobayashi, A., \& Kawasaki, C. (2005). Heart rate variability repetitive exposure to music. Biological Psychology. $70,61-66$.

Melo, G. A. A., Rodrigues, A. B., Firmeza, M. A., Grangeiro, A. S., Oliveira, P. P., \& Caetano, J. A. (2018).Musical intervention on anxiety and vital parameters of chronic renal patients: a randomized clinical trial. Rev. Latino-Am. Enfermagem. 26:e2978. http://dx.doi.org/10.1590/1518-8345.2123.2978

Nieto-Romero, R. M. (2017). Efectos de la musicoterapia sobre el nivel de ansiedad del adulto cardiópata sometido a resonancia magnética. Enfermería Universitaria. 14(2):88-96.

Nogueira, M. L., Garner, D. M., Osório, E., de Abreu, L. C., \& Vitor Engrácia Valenti, V. E. (2015). Globally chaotic analysis of Heart Rate Variability during acute auditory stimulus by heavy metal music. MedicalExpress. 2(5):M150504.

Paschoal, M. A., Volnti V. M., Pires, C. S., \& Fernandes, F. C. (2006). Variabilidade da freqüência cardíaca em diferentes faixas etárias. Rev Bras Fisioter. $10(4), 413-9$.

Pereira, A. S., Shitsuka, D. M., Parreira, F. J \& Shitsuka, R. (2018). Metodologia da pesquisa cientifica. UFSM https://repositorio.ufsm.br/bitstream/handle/1/15824/Lic_Computacao_Metodologia-Pesquisa-Cientifica.pdf?sequence=1

Peretz, I., Blood, A. J., Penhune, V., \& Zatorre, R. (2001). Cortical deafness to dissonance. Brain. 124( 5), 928-40.

Santos, G. L. de S., Carvalho, E. T. de., Selva, O. (2020). Music in early childhood education as a tool in child's cognitive development. Research, Society and Development. 9 (7), e460974259. 10.33448/rsd-v9i7.4259.

Schuit, A. J., van Amelsvoort, L. G., Verheij, T. C., Rijneke, R. D., Maan, A. C., Swenne, C. A., \& Schouten, E. G. (1999). Exercise training and heart rate variability in older people. Med Sci Sports Exerc. 31(6), 816-21.

Silva, K. F., Felix, M. M., Cruz, L. F., Barichello, E., Pires, P. S., Mattia, A. L., Haas, V. J., Barbosa, M. H. (2021). Efeitos da música na ansiedade de doadores de sangue: ensaio clínico randomizado. Acta Paul Enferm. 34:eAPE00461.

Tshiswaka, S. K \& Pinheiro, S. L. (2020). Effect of music on reducing anxiety in children during dental treatment. RGO, Rev Gaúch Odontol. 68: e20200033. http://dx.doi.org/10.1590/1981-863720200003320190049

Zatorre, R. J., \& Salimpoor, V. N. (2013). From perception to pleasure: music and its neural substrates. Proc Natl Acad Sci U S A. 110 (2), $10430-7$.

Zuttin, R. S., Moreno, M. A., Cesár, M. C., Martins, L. E. B., Catai, A. M., \& Silva, E. (2008). Avaliação da modulação autonômica da frequência cardíaca nas posturas supina e sentada de homens jovens saudáveis. Rev Bras Fisioter. 12:7-12. 